\title{
A NOTE OF CORRECTION
}

\section{CHUAN-CHIH HSIUNG}

Professor L. A. Santalo has called my attention to errors in the metric characterizations (12) and (20) of my paper entitled Invariants of intersection of certain pairs of space curves, Bull. Amer. Math. Soc. vol. 55 (1949) pp. 623-628. The correction of these will be stated as follows.

1. In the first place, we consider the two curves $C, \bar{C}$ given by the expansions (1), (2). Through the origin 0 draw the $X^{\prime}$-axis in the $X Z$-plane perpendicular to the $Z$-axis, and then draw the $Y^{\prime}$-axis perpendicular to the $X^{\prime} Z$-plane. Let $\theta, \phi, \psi$ be respectively the angles between the $X-, X^{\prime}$-axes, the $Y$-, $Z$-axes, and the osculating planes $\tau, \bar{\tau}$. If $x^{\prime}, y^{\prime}, z^{\prime}$ are the coordinates of a point in space referred to the new orthogonal coordinate system $0=X^{\prime} Y^{\prime} Z$, then the expansions of the curves $C, \bar{C}$ in the neighborhood of the point 0 become

$C: y^{\prime}=-r \tan ^{3} \psi x^{\prime 3}+\cdots, z^{\prime}=\tan \theta x^{\prime}+a \tan ^{2} \psi x^{\prime 2}+\cdots ;$ $\bar{C}: x^{\prime}=\cot \psi y^{\prime}-\rho \cot \psi y^{\prime 3}+\cdots, \quad z^{\prime}=\csc \psi \cot \phi y^{\prime}+\alpha y^{\prime 2}+\cdots$.

Thus we can easily obtain the following metric characterization, instead of (12), of the invariant $I$ given by equation (6):

$$
I=-\frac{\cos ^{3} \theta}{\sin ^{8} \phi} \frac{R \bar{T}}{T \bar{R}} .
$$

2. We next consider the two curves $C, \bar{C}$ given by the expansions (15), (16). Without loss of generality we may assume the $Z$-axis to be perpendicular to the $Y$-axis. Through the origin 0 draw the $X^{\prime}$-axis perpendicular to the $Y Z$-plane. Let $\omega, \theta$ be respectively the angles between the $X$-, $Y$-axes, and the osculating planes $\tau, \bar{\tau}$. If $x^{\prime}, y^{\prime}, z^{\prime}$ are the coordinates of a point in space referred to the new orthogonal coordinate system $0=X^{\prime} Y Z$, then the expansions of the curves $C, \bar{C}$ in the neighborhood of the point 0 become

$$
\begin{aligned}
C: z^{\prime} & =\frac{1}{\tan \theta}\left(x^{\prime}-r x^{\prime 3}\right)+\cdots, \\
y^{\prime} & =\frac{1}{\sin \theta \tan \omega}\left(x^{\prime}-a x^{\prime 2}\right)+\cdots ; \\
\bar{C}: x^{\prime} & =-\rho \sin ^{3} \theta \tan ^{3} \omega y^{\prime 3}+\cdots,
\end{aligned}
$$

Received by the editors February 11, 1950. 


$$
z^{\prime}=-\alpha \sin \theta \cos \theta \tan ^{2} \omega y^{\prime 2}+\cdots .
$$

Thus we can easily obtain the following metric characterization, instead of (20), of the invariant $J$ given by equation (19):

$$
J=\left(\frac{2 \sin \omega}{3 \sin \theta}\right)^{6} \frac{R^{2} \bar{R}^{4}}{T^{5} \bar{T}} .
$$

The UNIVERSITY OF Wisconsin

\section{A REMARK ABOUT OUR NOTE "TRANSCENDENCE OF FACTORIAL SERIES WITH PERIODIC COEFFICIENTS"1}

VERNE E. DIETRICH AND ARTHUR ROSENTHAL

Dr. B. McMillan has called our attention to his paper $A$ note on transcendental numbers, Journal of Mathematics and Physics vol. 18 (1939) pp. 28-33. Moreover, Prof. C. D. Olds has referred us to the article On transcendental numbers by T. Itihara and K. Ôishi, Tôhoku Math. J. vol. 37 (1933) pp. 209-221. We had not known both these papers, which are closely related to our result. In the meantime Prof. J. Popken, in his review of our note (Mathematical Reviews vol. 11 (1950) p. 331), also called attention to B. McMillan's general theorem II, but remarked: "However, McMillan's proof is not quite correct and his results need supplementing." (Concerning this see a forthcoming paper of Prof. J. Popken.) On the other hand our result does not formally follow from T. Itihara's Theorem 1 (which admits $k$ exceptional values). But our result can indeed easily be obtained also by the method applied in the proofs of T. Itihara's Theorem 1 or B. McMillan's Theorem II. However, our method is different and more direct.

Purdue University

Received by the editors October 18, 1951.

1 Bull. Amer. Math. Soc. vol. 55 (1949) pp. 954-956. 